\title{
Review
}

\section{Is there a place for liver transplantation in cases with unresectable central cholangiocarcinoma?}

\author{
Nicolae Bacalbașa ${ }^{1,2,3}$, Irina Balescu ${ }^{4}$, Mihaela Vilcu ${ }^{2,3}$, Iulian Brezean ${ }^{2,3}$, Alin Bodog ${ }^{5}$ \\ ${ }^{1}$ Center of Excellence in Translational Medicine, Fundeni Clinical Institute, Bucharest, Romania \\ ${ }^{2}$ Carol Davila University of Medicine and Pharmacy, Bucharest, Romania \\ ${ }^{3}$ Ion Cantacuzino Clinical Hospital, Bucharest, Romania \\ ${ }^{4}$ Ponderas Academic Hospital, Bucharest, Romania \\ ${ }^{5}$ Oradea Medical University, Oradea, Romania
}

\begin{abstract}
Central cholangiocarcinoma represents a very aggressive malignancy associated with poor survival rats especially due to the fact that most patients are diagnosed in advanced stages of the disease when curative resection is no longer feasible. In order to try improving the long-term outcomes of these patients, especially in unresectable cases liver transplantation has been proposed with promising results. This is a literature review of the largest studies focused on this subject.

Cholangiocarcinomas remain aggressive tumors with poor outcomes whenever surgery with curative intent is not feasible. Currently, the largest experience accumulated consists of neoadjuvant external beam radiotherapy in association with beam radiation, 5 fluorouracil and capecitabine followed by orthotopic liver transplantation. Following this therapeutic protocol excellent long-term outcomes have been reported even if vascular involvement is present.
\end{abstract}

\footnotetext{
Keywords $\quad:$ unresectable, central cholangiocarcinoma, liver transplantation

Highlights $\quad \checkmark$ Current attitude for cholangiocarcinomas is represented by neoadjuvant external beam radiotherapy, 5 fluorouracil and capecitabine, followed by orthotopic liver transplantation.

$\checkmark$ The presence of peritoneal, lymph node, intrahepatic or extrahepatic metastases is associated with significantly poorer outcomes, being generally considered a contraindication for liver transplantation.
}

To cite this article: Bacalbașa N, Balescu I, Vilcu M, Brezean I, Bodog A. Is there a place for liver transplantation in cases with unresectable central cholangiocarcinoma? J Clin Invest Surg. 2018; 3(2): 57-61. DOI: 10.25083/2559.5555/3.2/57.61

$\triangle$ *Corresponding author: Nicolae Bacalbasa, Carol Davila University of Medicine and Pharmacy, Bucharest, Romania; E-mail: nicolae_bacalbasa@yahoo.ro 


\section{Introduction}

Cholangiocarcinomas account for up to $10 \%$ of all primary liver malignancies and less than $3 \%$ of all gastrointestinal malignant tumors and originate from biliary tract epithelium or hepatic progenitor cells (1). Among liver malignancies, cholangiocarcinomas range for the second most common hepatic neoplastic disease, following hepatocarcinomas; however, their incidence reports a high rate of variation among different populations due to the different exposure to the main risk factors $(2,3)$. Similarly to other biliary tree malignant tumors, cholangiocarcinomas are associated with increasing age, presenting a peak of incidence between 55 and 74 years old as well as with the male sex (4). Other risk factors for developing cholangiocarcinomas are represented by exposure to Thorotrast, Caroli's disease, hepatolithiasis, metabolic syndrome and bile duct cysts $(5,6)$.

When it comes to their clinical course, cholangiocarcinomas are recognized as having an aggressive biological behavior, associated with poor outcomes whenever surgery with curative intent is not feasible; therefore, is considered that only $25 \%$ of cases are amenable to radical surgery while the overall survival ranges between $25-30 \%(7,8)$. Moreover, surgery is associated with a significant rate of postoperative complications and, consequently with a high morbidity rate (9).

Mainly, it is considered that there are two subtypes of cholangiocarcinomas: de novo lesions, diagnosed in patients with no previous biliary pathology and they usually develop in the hilar region and secondary lesions, developed on pre-existent biliary diseases such as primary sclerosing cholangitis (10-12). In the second eventuality the overall prognostic is even scarcer $(9,12)$. According to the anatomical localization, cholangiocarcinomas are classified into three categories: intrahepatic lesions (accounting for $15-20 \%$ of cases), perihilar lesions (accounting for $60-70 \%$ of cases) and distal lesions (representing 20-30\% of cases). According to this classification the three categories present certain common features but also important differences responsible for the particular outcomes of each lesion (13). However, the most recent classification from the World Health Organization (WHO) and the Union for International Cancer Control (UICC) refers only to two anatomical categories: intrahepatic oncological therapies followed by transplantation was cholangiocarcinomas (developing from the second order reconsidered (12). Interestingly, certain authors report bile ducts and the more proximal bile ducts) and that patients with hilar cholangiocarcinomas have a

extrahepatic lesions (including lesions originating from the two main hepatic bile ducts - Klatskin tumors and more distal tumors originating from the common bile duct but excluding Vater's ampulla tumors) $(14,15)$.

\section{Discussions}

- Principles of surgery in cholangiocarcinomas The main objective of the surgical approach of cholangiocarcinomas is represented by achieving a radical resection; this goal is achieved if the margins' status is R0 (no residual tumor). Other possibilities after surgery are represented by $\mathrm{R} 1$ resection (microscopic remnant tumoral volume) or $\mathrm{R} 2$ resection (macroscopic residual tumoral volume). However, it seems that only cases benefiting from $\mathrm{R} 0$ resection report a significant improvement of the overall survival; therefore, is considered that only $20 \%$ of cases diagnosed with cholangiocarcinomas are candidates for resection with curative intent (1). Moreover, even if curative resection is feasible, another important aspect which should be taken in consideration is related to the remnant liver volume; although in certain cases radical resection with curative intent is technically feasible, if the remnant volume of the liver does not exceed $30 \%$ of the total volume of the liver postoperative organ failure risk is significant, therefore is considered that surgery should not be performed (1).

- Therapeutic strategies in unresectable cholangiocarcinomas

Once liver transplantation was proved to be an effective therapeutic strategy for hepatic carcinomas, the method was also proposed for cases with cholangiocarcinomas from the early 60's in order to improve the overall outcomes of these patients. However, the initial results were rather discouraging, with increased rates of relapse; for example, the Cincinnati Transplant Tumor Registry reported a 5-year survival rate after transplantation of $28 \%$ and a $51 \%$ rate of recurrence, the most commonly reported relapses being reported within the first two years at the level of the liver graft (in $47 \%$ of cases) and lungs respectively (in $30 \%$ of cases) (16). In consequence, the diagnostic of cholangiocarcinoma became a contraindication for liver transplantation in a significant number of centers (17). However, later on the oncologic therapies such as radiotherapy and chemotherapy reported a significant 58 
poorer outcome after neoadjuvant chemo-radiation presence of metastatic lesions was excluded so the followed by orthotopic liver transplantation when patients were further submitted to liver transplantation; compared with patients who develop the authors reported a 5-year survival rate after cholangiocarcinomas on pre-existent biliary diseases transplantation of $71 \%$ (20).

(12). These outcomes might be explained through the Another interesting therapeutic strategy was fact that performing liver transplantation in a patient presented by Lunsford et al. in the study presented in with pre-existent biliary disease will eliminate other The Lancet Gastroenterology and Hepatology, in 2018 potential malignant transformations originating from the (21). The study is a prospective one and included bile ducts; in the meantime, it will entirely replace the patients with unresectable cholangiocarcinomas without hepatic parenchyma, which might had been the theatre extrahepatic or vascular involvement who were for recurrent biliary tree infections (18).

It has been more than 25 years since the researchers from Mayo Clinic proposed an innovative therapeutic protocol in order to maximize the long-term outcomes for the patients with unresectable central cholangiocarcinomas. The protocol consisted of administration of external beam radiotherapy (up to 45Gy) delivered over three weeks followed by a single dose of transcatheter brachytherapy (up to 30Gy) and concomitant 5-fluorouracil administration. After ending this protocol oral capecitabine was given in all cases until orthotopic liver transplantation was possible (19). From the year of 2000, when the first results were reported, researchers from Mayo Clinic presented excellent results after performing this technique, with overall survival rates of $92 \%, 81 \%$ and $74 \%$ at $1,3-$ and 5-year follow-up (9). These data seem to be also the result of an improved perioperative outcome, with significantly lower rates of perioperative deaths when compared to other similar studies (12).

One of the largest studies published by the researchers from Mayo Clinic regarding the role of liver transplantation in cholangiocarcinomas included 90 patients submitted to neoadjuvant chemo-irradiation followed by liver transplantation after 1993. The presence of vascular involvement wasn't considered as submitted to neoadjuvant chemotherapy (gemcitabinecisplatin or gemcitabine-capecitabine protocol) followed by liver transplantation if they reported a radiological stabilization of the disease within six months. The study included six such patients who benefitted from liver transplantation after a median duration from diagnostic of 26 months, all patients being submitted to neoadjuvant chemotherapy while awaiting liver transplant. After a median follow-up interval of 26 months three cases developed recurrent disease; the overall survival rate was $100 \%$ at 1 year, $83.3 \%$ at 3 years and $83.3 \%$ at 5 years, demonstrating that in selected cases a significant benefit in terms of survival can be expected (21).

- Factors influencing the overall outcomes after liver transplantationfor unresectable cholangiocarcinomas An interesting subject for patients included in this therapeutic strategy is related to the influence of the time spent on the waiting list before transplantation and the overall outcomes; in the study conducted by Duignan et al. the authors reported a surprising finding, that there was no correlation between the time spent on waiting for liver transplantation and the long-term outcomes; moreover, the authors underlined the fact that the two patients reporting the longer survival spent more than exclusion criteria; contrarily, the presence of underlined the fact that the only significant prognostic intrahepatic or extrahepatic metastases as well as the factor was related to the absence of residual tumor at the association of gallbladder involvement was considered end of liver transplantation. Due to the fact that this as contraindications. Similarly, to the previous study, factor cannot be assessed during the preoperative the neoadjuvant therapy included 4000 to $4500 \mathrm{cGy}$ of workup (being considered that it can be correctly external radiation therapy followed by 2000 to $3000 \mathrm{cGy}$ estimated only after ending the phase of liver resection) of transcatheterial irradiation and concomitant 5- the authors went further and studied whether there can fluorouracil while capecitabine was administrated until be established a connection between the preoperative liver transplantation. Moreover, before performing levels of CA 19-9 and the completeness of resection; orthotopic liver transplantation, in all cases a staging they concluded that a higher than 100 levels of CA 19-9 laparotomy including serial biopsies from the regional is strongly predictable for the presence of residual tumor lymph nodes, peritoneum was performed. The study after liver resection (12). Similar results in regard with initially included 148 patients; however, after CA 19-9 levels and the overall survival was reported by performing the staging laparotomy only in 90 cases the the researchers from Mayo Clinic (22). 
Another study published by Heimbach et al. at Mayo Clinic demonstrated that the most important factors negatively influencing survival were represented by older age, prior cholecystectomy, CA 19-9 level higher than 100 at the moment of transplantation, presence of visible mass at imaging and prolonged waiting time ; other factors influencing the recurrence risk were represented by residual lesions in the explanted liver larger than $2 \mathrm{~cm}$, higher tumoral grades as well as the presence of perineural invasion (23).

\section{Conclusions}

Cholangiocarcinomas remain aggressive tumors with poor outcomes whenever surgery with curative intent is not feasible. The option of liver transplantation, initially proposed in the early 60's which had been initially considered as inefficient due to the high rate of relapse, was reconsidered later on in association with perioperative oncologic treatment. Nowadays the largest experience originates from the Mayo Clinic and consists of neoadjuvant external beam radiotherapy in association with beam radiation, 5 fluorouracil and capecitabine followed by orthotopic liver transplantation. Following this therapeutic protocol excellent long-term outcomes have been reported even if vascular involvement is present. However, the presence of peritoneal, lymph node, intrahepatic or extrahepatic metastases is associated with significantly poorer outcomes and therefore is considered as a formal contraindication for liver transplantation.

\section{Conflict of interest disclosure}

There are no known conflicts of interest in the publication of this article, and there was no financial support that could have influenced the outcomes. The manuscript was read and approved by all authors.

\section{Compliance with ethical standards}

Any aspect of the work covered in this manuscript that has involved human patients has been conducted with the ethical approval of all relevant bodies and that such approvals are acknowledged within the manuscript.

\section{References}

1. Hoyos S, Navas MC, Restrepo JC, Botero RC. Current controversies in cholangiocarcinoma. Biochim Biophys Acta Mol Basis Dis. 2018; 1864(4): 1461-67. PMID: 28756216 DOI: 10.1016/j.bbadis.2017.07.027

2. International Agency for Research on Cancer (IARC), GLOBOCAN 2012: Estimated Cancer
Incidence, Mortality and Prevalence Worldwide in 2012. http://globocan.iarc.fr/Default.aspx

3. Khan SA, Toledano MB, Taylor-Robinson SD. Epidemiology, risk factors, and pathogenesis of cholangiocarcinoma. HPB (Oxford). 2008; 10: 7782. PMID: 18773060 , DOI: 10.1080/13651820801992641

4. Shaib YH, Davila JA, McGlynn K, El Serag HB. Rising incidence of intrahepatic cholangiocarcinoma in the United States: a true increase? J Hepatol. 2004; 40(3): 472-77. PMID: 15123362, DOI: 10.1016/j.jhep.2003.11.030

5. Lazaridis KN, LaRusso NF. The Cholangiopathies. Mayo Clin Proc. 2015; 90(6): 791-800. PMID: 25957621, DOI: 10.1016/j.mayocp.2015.03.017

6. Banales JM, Cardinale V, Carpino G et al. Expert consensus document: Cholangiocarcinoma: current knowledge and future perspectives consensus statement from the European Network for the Study of Cholangiocarcinoma (ENS-CCA). Nat Rev Gastroenterol Hepatol. 2016; 13(5): 261-80. PMID: 27095655, DOI: 10.1038/nrgastro.2016.51

7. Silva MA, Tekin K, Aytekin F, Bramhall SR, Buckels JA, Mirza DF. Surgery for hilar cholangiocarcinoma; a 10 year experience of a tertiary referral centre in the UK. Eur J Surg Oncol. 2005; 31(5): 533-39. PMID: 15922889, DOI: 10.1016/j.ejso.2005.02.021

8. Burke EC, Jarnagin WR, Hochwald SN, Pisters PW, Fong Y, Blumgart LH. Hilar Cholangiocarcinoma: patterns of spread, the importance of hepatic resection for curative operation, and a presurgical clinical staging system. Ann Surg. 1998; 228(3): 385-94. PMID: 9742921

9. Rosen CB, Darwish MS, Heimbach JK, Nyberg SL, Nagorney DM, Gores GJ. Neoadjuvant therapy and liver transplantation for hilar cholangiocarcinoma: is pretreatment pathological confirmation of diagnosis necessary? J Am Coll Surg. 2012; 215(1): 31-8. PMID: 22621893, DOI: 10.1016/j.jamcollsurg.2012.03.014

10. Klatskin G. Adenocarcinoma of the hepatic duct at its bifurcation within the porta hepatis. An usual tumor with distinctive clinical and pathological features. Am J Med. 1965; 38: 241-56.

11. Blechacz B, Gores GJ. Cholangiocarcinoma: advances in pathogenesis, diagnosis, and treatment. Hepatology. 2008; 48(1): 308-21. PMID: 18536057 DOI: $10.1002 /$ hep. 22310 
12. Duignan S, Maguire D, Ravichand CS et al. 19. De VI, Steers JL, Burch PA et al. Prolonged Neoadjuvant chemoradiotherapy followed by liver transplantation for unresectable cholangiocarcinoma: a single-centre national experience. HPB (Oxford). 2014; 16: 91-98.

disease-free survival after orthotopic liver transplantation plus adjuvant chemoirradiation for cholangiocarcinoma. Liver Transpl. 2000; 6: 30916.

13. DeOliveira ML, Cunningham SC, Cameron JL et al. 20. Rosen CB, Heimbach JK, Gores GJ. Surgery for Cholangiocarcinoma: thirty-one-year experience with 564 patients at a single institution. Ann Surg. 2007; 245(5): 755-62. PMID: 17457168, DOI: 10.1097/01.sla.0000251366.62632.d3

cholangiocarcinoma: the role of liver transplantation. HPB (Oxford). 2008; 10(3): 18689. PMID: 18773052

DOI: $10.1080 / 13651820801992542$

14. Bosman FT, Carneiro F, Hruban RH, Theise ND. 21. Lunsford KE, Javle M, Heyne K et al. Liver WHO Classification of Tumours of the Digestive System, Fourth edition, 2010.

15. Brierley JD, Gospodarowicz MK, Wittekind C. TNM Classification of Malignant Tumours, $7^{\text {th }}$ edition, Seventh, 2011.

transplantation for locally advanced intrahepatic cholangiocarcinoma treated with neoadjuvant therapy: a prospective case-series. Lancet Gastroenterol Hepatol. 2018; 3(5): 337-48. PMID: 29548617, DOI: 10.1016/S2468-1253(18)30045-1

16. Meyer CG, Penn I, James L. Liver transplantation 22. Rea DJ, Rosen CB, Nagorney DM, Heimbach JK, for cholangiocarcinoma: results in 207 patients. Transplantation. 2000; 69(8): 1633-37. PMID: 10836374

17. Khan SA, Davidson BR, Goldin R et al. Guidelines for the diagnosis and treatment of cholangiocarcinoma: consensus document. Gut. 2002; 51 Suppl 6: VI1-VI9.

18. Zhu JQ, He Q, Li XL. Liver transplantation benefits selected patients with hilar cholangiocarcinoma and colorectal liver metastases. Hepatobiliary Pancreat Dis Int. 2018; 17: 385-86. Gores GJ. Transplantation for cholangiocarcinoma: when and for whom? Surg Oncol Clin N Am. 2009; 18(2): 325-37, ix. PMID: 19306815, DOI: 10.1016/j.soc. 2008.12 .008

23. Heimbach JK, Gores GJ, Haddock MG et al. Predictors of disease recurrence following neoadjuvant chemoradiotherapy and liver transplantation for unresectable perihilar cholangiocarcinoma. Transplantation. 2006; 82(12): 1703-7. PMID: 17198263 